\title{
Fusing Landmark-based Features at Kernel Level for Face Recognition
}

\author{
Ke-Kun Huanga, ${ }^{\mathrm{a}, \mathrm{b}}$ Dao-Qing Dai ${ }^{\mathrm{b}, *}$, Chuan-Xian Ren ${ }^{\mathrm{b}}$, Yu-Feng Yu ${ }^{\mathrm{b}}$, \\ Zhao-Rong Laic,b \\ ${ }^{a}$ School of Mathematics, JiaYing University, Meizhou, Guangdong, 514015, China. \\ ${ }^{b}$ Intelligent Data Center and Department of Mathematics, Sun Yat-Sen University, Guangzhou \\ 510275, China. \\ ${ }^{c}$ Department of Mathematics, JiNan University, Guangzhou 510632, China.
}

\begin{abstract}
Because of the dramatic intra-class variations in lighting, expression and pose of face images, no single feature is rich enough to capture all the discriminant information, fusing multiple features is an efficient way to improve performance for face recognition. But most of existing fusing methods use features sampling at fixed gird and manually set too many parameters, thus their performances are limited. In this paper, we first propose an improved landmark-based multi-scale LBP feature to address the dramatic pose and expression variations, which samples features around landmarks instead of fixed grid. Then we propose a novel model which fuses LBP feature and Gabor feature at kernel-level to capture the information of facial texture and facial shape, where the weighted coefficients

\footnotetext{
This work is supported in part by National Science Foundation of China under Grants 61203248, 11171354, 90920007, 61375033, 61403164 and 61572536.

*Corresponding author

Email addresses: kkcocoon@163. com (Ke-Kun Huang), stsddq@mail.sysu.edu. cn (Dao-Qing Dai), rchuanx@mail.sysu.edu.cn (Chuan-Xian Ren), yuyufeng220@163. com (Yu-Feng Yu), laizhr@mail2. sysu.edu. cn (Zhao-Rong Lai)
} 
between kernels, the discriminant projection matrix and the standard deviations of RBF kernel are simultaneously learnt by the proposed optimization algorithm. Experiments are done on LFW, AR and Extended Yale B datasets, and results show that not only does the proposed method get much better identification performance than some state-of-the-art methods, but it also achieves competitive result for verification task.

Keywords: Face recognition, Landmark-based feature, Feature fusion, Multi-kernel discriminant analysis, Face identification, Face verification. 


\section{Introduction}

Face recognition has attracted much attention because of its wide range of applications in access control and video surveillance [1]. Because of the dramatic intra-class variations in facial expression, viewing angle, lighting conditions, occlusions and image quality, face recognition is still a challenging problem. One of the key important issue is to find some efficient features that are both discriminative for inter-class difference and invariant to intra-class variations. Because no single feature is rich enough to capture all the discriminative information, fusing complementary features has become an active research topic in face recognition $[2,3,4]$.

To fuse multiple features, we first need to find some efficient features. In the past decades, many feature extraction methods have been proposed for face recognition. Local Binary Patterns (LBP), introduced by Ojala et al.[5] in 1996, is a good choice for coding fine details of facial appearance and texture. They later extend LBP to multiple scales and compress the LBP codes by the uniform patterns [6]. Because LBP can capture small texture details and is resistant to lighting changes, Ahonen et al. introduce an LBP based method for face recognition [7], which divides the face into a regular grid of cells and calculates the histogram of the uniform LBP's within each cell. Finally, the cell-level histograms are concatenated to produce a global descriptor vector. For LBP's impressive performance [8], many its variants have been proposed for face recognition $[1,9,10]$.

Besides LBP, Gabor transform based feature extraction methods also have been an active research area in recent years. Gabor wavelets capture the local structure corresponding to specific spatial frequency, spatial locality, and selective orientation. Gabor feature has been demonstrated to be discriminative and 
robust to illumination and expression changes. Liu et al.[11] introduce a GaborFisher classifier for face recognition. Shen et al. [12] present the recognition results for Gabor features and general discriminant analysis. Histogram of Gabor phase feature is proposed by Zhang et al. [13] for face recognition. Xie et al. [14] propose learned local Gabor patterns for face representation and recognition.

There are other features that can be used in face recognition, such as Gradientface [15], Scale Invariant Feature Transform (SIFT) [16], Histograms of Oriented Gradients (HOG) [17] and LEarning-based descriptor (LE) [18]. Although these features have shown promising performances on public face datasets collected in the controlled environment, these hand-crafted descriptors cannot work well for uncontrolled face recognition. Chen et al. [19] densely sample multi-scale descriptors centered at dense facial landmarks after accurate face alignment. For uncontrolled face verification, the resulting high-dimensional features achieve significant improvements over their low-dimensional version sampled at fixed grid.

Though the above mentioned features have been demonstrated their effectiveness respectively, for most of fusing models, combing all the features cannot get better performance than only combing a few features, but introduces much more computational cost. So, selecting some appropriate and complementary features is crucial for fusing model to get good performance. We find that LBP feature and Gabor feature are complementary because LBP captures small appearance details while Gabor feature reflects the facial shape. So we consider fusing the two types of features. In addition, because landmark-based feature can deal with the misaligned situation in face image better than grid-based feature, so we consider landmark-based feature for face recognition.

After selecting appropriate features, it is important to build a good fusing mod- 
el. Because Kernel Discriminant Analysis (KDA) [20] can project the data onto a non-linear discriminant subspace to suppress intra-class variations and maximize the gap between the images from different persons, many KDA-based fusing models have been proposed. Tan et. al. [2] apply kernel discriminative common vector for the combined feature vector to extract discriminant nonlinear features for recognition. Lin et al. [3] fuse diverse image descriptors by the proposed multiple kernel learning for dimensionality reduction. Ren et al. [4] present bandreweighed Gabor kernel embedding for face representation and recognition, using Multiple Orientation and Scale Transforms (MOST) with multiple Gabor band features. But the above methods set too many parameters manually, including the standard deviations of RBF kernel, thus their performances are limited. So we propose a novel model to fuse LBP feature and Gabor feature at kernel-level, where the weighted coefficients between kernels, the discriminant projection matrix and the standard deviations of RBF kernel are simultaneously learnt.

Most of papers concern only one sub-problem of face recognition, i.e., either face identification or face verification. Face verification is to determine whether a pair of face images are from the same person or not, which is a binary-class problem. But in traditional face identification, we must identify which person a face image belongs to, which is a multi-class problem. Face identification and face verification are two sub-problems in face recognition [21]. Though some methods can achieve impressive results in a sub-problem, they can't get high performance in another sub-problem. In contrast, our method concerns both sub-problems, and gets high performance in both sub-problems.

The main contributions of this paper are listed as follows:

- We propose an improved landmark-based multi-scale LBP feature, which 
adopts different scales of LBP and different sizes of patch to generate multi-scale features. To control the resulting feature dimension, we reduce the number of landmarks along with the increase of LBP scale. Because the landmark-based features maybe lose the geometric information among the landmarks, we also propose combining the fixed grid-based features to improve the performance.

- We propose a novel model to fuse LBP feature and Gabor feature at kernellevel, where the weighted coefficients between kernels, the discriminant projection matrix and the standard deviations of RBF kernel are simultaneously learnt.

- We propose an optimization algorithm to solve the kernel-level fusing model, which alternatively calculates the three types of parameters.

The remainder of the paper is organized as follows: In section 2, we describe the proposed method in detail. The experimental results are given in section 3 . Finally, we provide the conclusion in section 4.

\section{The proposed method}

In this section, we describe the proposed method in detail. In section 2.1, we propose an improved landmark-based multi-scale LBP feature. In section 2.2, we propose combining landmark-based feature and grid-based feature for face recognition. Then a novel kernel-level fusing model and corresponding optimization algorithm are proposed in section 2.4 and section 2.5 , respectively. 

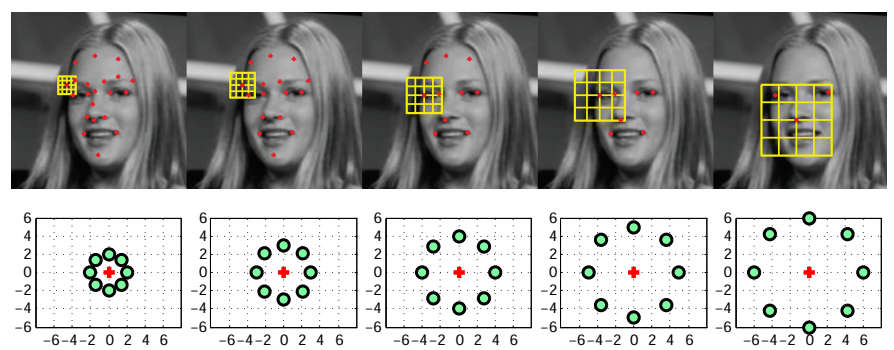

Figure 1: The proposed landmark-based multi-scale LBP feature. The uniform LBP codes are computed at 5 scales, using 8 sampling points on a circle of radius $2,3,4,5$ and 6 , respectively. Then a patch is extracted centering at each landmark of each scale, and is divided into $4 \times 4$ cells. Finally, we calculate the histogram of the LBP codes for each cell and concatenate all the histograms. Along with the increase of LBP scale, the number of landmarks is reduced, while the size of the patch is increased.

\subsection{An improved landmark-based multi-scale LBP feature}

Most of face recognition methods use grid-based feature [1] [7][11][22]. They extract some local features for a grid of locations in an aligned face image, and learn a subspace after concatenating all the local features. The features in the same location of two samples are compared with each other, no matter whether they are the same landmarks or not. Because of the dramatic variations for expression and pose, face images can hardly be aligned accurately. The grid-based methods may fail on the misaligned condition. To address this problem, Chen et al. propose the High Dimensional LBP (HDLBP) feature [19], which extracts LBP feature in some patches centering at each landmark positions. In the landmarkbased method, the features of the same landmarks of two samples are compared with each other, so HDLBP gets significant improvements for face verification over its low-dimensional version sampled at fixed grid. However, because the dimension of HDLBP is very high, it needs much computational and storage cost. In 
this subsection, we propose an improved landmark-based multi-scale LBP feature, denoted by LMLBP, to deal with this issue.

For each image, we first detect the facial landmark positions [23]. Then we apply similarity transformation using the landmark points to transform a face to a canonical frame, with size of $400 \times 400$. After landmark detection and face normalization, we extract 5 scales of uniform LBP feature. To be specific, the LBP code of a pixel $g_{0}$ is attained by the following equation:

$$
L B P_{R}=\sum_{p=0}^{7} t\left(g_{p}-g_{0}\right) 2^{p},
$$

where $g_{p}$ is the $p^{t h}$ pixel of 8 equally spaced pixels on a circle of radius $R$ centering at $g_{0}$. if $g>0$, then $t(g)=1$, otherwise $t(g)=0$. The original number of bins using 8 neighbors is 256 , which can be compressed to 59 by the uniform patterns[6]. We set $R=2,3,4,5,6$ for each scale, respectively, shown in the second row of Fig. 1.

Then we extract a patch centering at each landmark of each scale, and divide it into $4 \times 4$ cells. At the larger LBP scale $R$, the size of the patch $S_{R}$ are bigger, i.e.,

$$
S_{R}=S_{2}(\sqrt{2})^{R-2}
$$

where $S_{2}=40$ is the size of patch of the first LBP scale $(R=2)$. Note that at different LBP scales, the image sizes of are equal, while the patch sizes are different.

To control the resulting feature dimension, we reduce the number of landmarks along with the increase of LBP scale, shown in the first row of Fig. 1. Finally, we calculate the histogram of the uniform LBP codes for each cell and concatenate all the histograms to form the landmark-based multi-scale LBP feature, resulting 


\subsection{Combining landmark-based feature and grid-based feature}

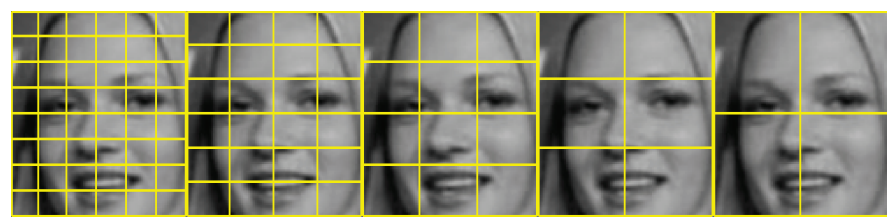

Figure 2: Grid-based multi-scale LBP feature. The image of each scale is devided into a grid of cells and extract LBP descriptor for each cell. Note that the sizes of the cells are bigger at the larger scale. 
Because the landmark-based features may lose the geometric information among the landmarks, we combine the grid-based multi-scale features to improve the performance. We crop a center region after transforming a face to a canonical frame. Similar to landmark-based feature, we first extract 5 scales of uniform LBP feature. Then we divide the LBP image of each scale into a grid of cells. At the larger LBP scale, the size of the cells are bigger, shown in the Fig 2. Finally, we calculate the histogram of the uniform LBP codes for each cell and concatenate all the histograms to form the grid-based multi-scale LBP feature.

After extracting the landmark-based multi-scale LBP feature and grid-based multi-scale LBP feature, we simply concatenate the two features at feature level to get the final LBP feature. We will show in the experiment that combining gridbased feature and landmark-based feature can get higher performance.

\subsection{Combining Gabor feature}

Gabor wavelets can capture a number of salient visual properties including spatial localization, orientation selectivity and spatial frequency selectivity quite well, which can encode facial shape and appearance information. They have been widely used in face recognition[11]. As many face recognition studies, we use 40 Gabor wavelets of 5 different scales and 8 orientations. By convolving the input image with these 40 Gabor kernels, we get 40 Gabor images.

To get more useful information, we resize the input image with a scaling factor of $1, \frac{1}{\sqrt{2}}, \frac{1}{2}, \frac{1}{2 \sqrt{2}}, \frac{1}{4}$, respectively, to get five input images. Then we extract 40 Gabor images for each input image, and normalize each Gabor image to zero mean and unit variance. We extract fixed-size patches centering at each landmark in each Gabor image of each input image. Similar to the previous subsection, to control the resulting feature dimension, we reduce the number of landmarks along 
with the decrease of the size of input image. Then we downsample each image patch by $4 \times 4$ uniform lattices. Finally, we concatenate all the down-sampled patches to form the landmark-based Gabor feature.

To get the grid-based Gabor feature, the 40 Gabor images are directly downsampled by $4 \times 4$ uniform grids. We construct a vector out of each downsampled responses, normalize it to zero mean and unit variance, and concatenate them into a single dimensional feature vector.

Similar to LBP feature extraction, after extracting the landmark-based Gabor feature and grid-based Gabor feature, we simply concatenate the two features at feature level to attain the final Gabor feature.

\subsection{A novel kernel-level fusing model}

In this subsection, we propose a novel kernel-level fusing model to fuse LBP and Gabor feature, where the weighted coefficients between kernels, projection matrix and kernel parameters are simultaneously learnt.

Suppose we have $N$ training samples, $\left\{\mathbf{x}_{i}\right\}_{i=1}^{N}, \mathbf{x}_{i}=\left\{\mathbf{L}_{i}, \mathbf{G}_{i}\right\}$, where $\mathbf{L}_{i}$ is the obtained LBP feature of $i^{\text {th }}$ training sample, and $\mathbf{G}_{i}$ is the obtained Gabor feature. Suppose there is an ensemble kernel $k$ generated by linearly combining the base kernels $\left\{k_{L}, k_{G}\right\}$ :

$$
k\left(\mathbf{x}_{i}, \mathbf{x}_{j}\right)=\beta_{L} k_{L}\left(\mathbf{x}_{i}, \mathbf{x}_{j}\right)+\beta_{G} k_{G}\left(\mathbf{x}_{i}, \mathbf{x}_{j}\right),
$$

where

$$
\begin{gathered}
k_{L}\left(\mathbf{x}_{i}, \mathbf{x}_{j}\right)=\exp \left(\frac{-\chi^{2}\left(\mathbf{L}_{i}, \mathbf{L}_{j}\right)}{\sigma_{L}^{2}}\right) \\
k_{G}\left(\mathbf{x}_{i}, \mathbf{x}_{j}\right)=\exp \left(\frac{-\left\|\mathbf{G}_{i}-\mathbf{G}_{j}\right\|_{2}^{2}}{\sigma_{G}^{2}}\right)
\end{gathered}
$$


Note that we use chi-square distance for LBP feature and $l_{2}$ norm distance for Gabor feature to further exploit different advantages of different features. We will show in the experiments that the chi-square distance is significantly better than $l_{2}$ norm distance for LBP feature.

Let $\phi$ denote the non-linear mapping induced by the ensemble kernel $k$, where

$$
\left\langle\phi\left(\mathbf{x}_{i}\right), \phi\left(\mathbf{x}_{i}\right)\right\rangle=k\left(\mathbf{x}_{i}, \mathbf{x}_{j}\right) .
$$

Kernel based subspace methods can be exploited here as the basic criterion for feature extraction[20]. The objective function of KDA is:

$$
\max _{\mathbf{A}} \frac{\operatorname{Tr}\left(\mathbf{A}^{T} \mathbf{K W K A}\right)}{\operatorname{Tr}\left(\mathbf{A}^{T} \mathbf{K K A}\right)},
$$

where $\mathbf{K}$ is the kernel matrix, $\mathbf{K}_{i, j}=k\left(\mathbf{x}_{i}, \mathbf{x}_{j}\right)=\beta_{L} k_{L}\left(\mathbf{x}_{i}, \mathbf{x}_{j}\right)+\beta_{G} k_{G}\left(\mathbf{x}_{i}, \mathbf{x}_{j}\right)$, and $\mathbf{W}$ is defined as:

$$
\mathbf{W}_{i, j}=\left\{\begin{array}{l}
1 / m_{c}, \text { if } \mathbf{x}_{i} \text { and } \mathbf{x}_{j} \text { belong to the } c \text {-th class } \\
0, \text { otherwise. }
\end{array}\right.
$$

where $m_{c}$ is the number of samples of class $c$.

Besides the projection matrix $\mathbf{A}$, it is also important to find the optimal fusing weights $\beta=\left[\beta_{L}, \beta_{G}\right]^{T}$ and the kernel parameters $\sigma=\left[\sigma_{L}, \sigma_{G}\right]^{T}$. Based on Eq. (7), we propose a new model to fuse LBP feature and Gabor feature, which finds the kernel-level fusing weights, the kernel parameters and the projection matrix, to suppress intra-class variations and maximize the gap between the images from different persons, i.e.:

$$
\max _{\mathbf{A}, \boldsymbol{\beta}, \boldsymbol{\sigma}} \frac{\operatorname{Tr}\left(\mathbf{A}^{T}\left(\sum_{i, j=1}^{N} \mathbf{W}_{i, j} \mathbf{K}_{(i)} \boldsymbol{\beta} \boldsymbol{\beta}^{T} \mathbf{K}_{(j)}^{T}\right) \mathbf{A}\right)}{\operatorname{Tr}\left(\mathbf{A}^{T}\left(\sum_{i, j=1}^{N} \mathbf{K}_{(i)} \boldsymbol{\beta} \boldsymbol{\beta}^{T} \mathbf{K}_{(j)}^{T}\right) \mathbf{A}\right)},
$$


193 where $\mathbf{K}_{(i)}=\left[\begin{array}{cc}k_{L}\left(\mathbf{x}_{1}, \mathbf{x}_{i}\right) & k_{G}\left(\mathbf{x}_{1}, \mathbf{x}_{i}\right) \\ \ldots & \ldots \\ k_{L}\left(\mathbf{x}_{N}, \mathbf{x}_{i}\right) & k_{G}\left(\mathbf{x}_{N}, \mathbf{x}_{i}\right)\end{array}\right]$ and $\boldsymbol{\beta} \geq \mathbf{0}$.

\subsubsection{Optimize $\beta$}

When $\mathbf{A}$ and $\sigma$ are fixed, the objective function becomes:

$$
\max _{\boldsymbol{\beta}} \frac{\beta^{T} \mathbf{S}_{b}^{\mathbf{A}} \boldsymbol{\beta}}{\beta^{T} \mathbf{S}_{t}^{\mathbf{A}} \boldsymbol{\beta}}
$$

\footnotetext{
${ }^{1}$ Matlab code: http://www.cad.zju.edu.cn/home/dengcai/Data/DimensionReduction.html
} 
where $\mathbf{S}_{b}^{\mathbf{A}}=\sum_{i, j=1}^{N} w_{i, j} \mathbf{K}_{(i)}^{T} \mathbf{A} \mathbf{A}^{T} \mathbf{K}_{(j)}$ and $\mathbf{S}_{t}^{\mathbf{A}}=\sum_{i, j=1}^{N} \mathbf{K}_{(i)}^{T} \mathbf{A} \mathbf{A}^{T} \mathbf{K}_{(j)}$. The additional constraints $\beta \geq 0$ cause that the optimization problem can't be formulated as a generalized eigenvalue problem. It becomes a non-convex quadratically constrained quadratic programming problem which is known to be hard to solve. So we consider solving its convex relaxation by adding an auxiliary matrix B:

$$
\begin{gathered}
\max _{\boldsymbol{\beta}, \mathbf{B}} \operatorname{Tr}\left(\mathbf{S}_{b}^{\mathbf{A}} \mathbf{B}\right) \\
\text { s.t. }\left\{\begin{array}{c}
\operatorname{Tr}\left(\mathbf{S}_{t}^{\mathbf{A}} \mathbf{B}\right)=1 \\
\left(\begin{array}{cc}
1 & \boldsymbol{\beta} \\
\beta & \mathbf{B}
\end{array}\right) \succeq 0 . \\
\beta \geq \mathbf{0}
\end{array}\right.
\end{gathered}
$$

\subsubsection{Optimize $\sigma$}

When $\mathbf{A}$ and $\beta$ are fixed, the objective function becomes

$$
J(\boldsymbol{\sigma}) \doteq \frac{\operatorname{Tr}\left(\mathbf{A}^{T} \mathbf{K W K A}\right)}{\operatorname{Tr}\left(\mathbf{A}^{T} \mathbf{K K \mathbf { A }}\right)},
$$

To achieve the maximum of $J(\sigma)$, we propose an iterative method based on gradient descent. The kernel parameters $\sigma$ are updated along the direction of

\footnotetext{
${ }^{2}$ Matlab code: http://cvxr.com/cvx/
} 
maximizing the objective function:

$$
\boldsymbol{\sigma}_{i+1}=\boldsymbol{\sigma}_{i}-\rho \nabla J\left(\boldsymbol{\sigma}_{i}\right)
$$

where

$$
\begin{aligned}
& \nabla J(\boldsymbol{\sigma})=\left[\frac{\partial J}{\sigma_{L}}, \frac{\partial J}{\sigma_{G}}\right]^{T} \\
& \frac{\partial J}{\partial \sigma_{L}}=\frac{\operatorname{Tr}\left(\frac{\partial\left(\mathbf{A}^{T} \mathbf{K W K A}\right)}{\partial \sigma_{L}}\right) \operatorname{Tr}\left(\mathbf{A}^{T} \mathbf{K} \mathbf{K A}\right)}{\left(\operatorname{Tr}\left(\mathbf{A}^{T} \mathbf{K} \mathbf{K} \mathbf{A}\right)\right)^{2}} \\
& -\frac{\operatorname{Tr}\left(\mathbf{A}^{T} \mathbf{K} \mathbf{W K A}\right) \operatorname{Tr}\left(\frac{\partial\left(\mathbf{A}^{T} \mathbf{K K A}\right)}{\partial \sigma_{L}}\right)}{\left(\operatorname{Tr}\left(\mathbf{A}^{T} \mathbf{K K A}\right)\right)^{2}}, \\
& \operatorname{Tr}\left(\frac{\partial\left(\mathbf{A}^{T} \mathbf{K W K A}\right)}{\partial \sigma_{L}}\right)=\operatorname{Tr}\left(\mathbf{A}^{T} \frac{\partial \mathbf{K}}{\partial \sigma_{L}} \mathbf{W K A}+\mathbf{A}^{T} \mathbf{K} \mathbf{W} \frac{\partial \mathbf{K}}{\partial \sigma_{L}} \mathbf{A}\right) \\
& \operatorname{Tr}\left(\frac{\partial\left(\mathbf{A}^{T} \mathbf{K} \mathbf{K} \mathbf{A}\right)}{\partial \sigma_{L}}\right)=\operatorname{Tr}\left(\mathbf{A}^{T} \frac{\partial \mathbf{K}}{\partial \sigma_{L}} \mathbf{K} \mathbf{A}+\mathbf{A}^{T} \mathbf{K} \frac{\partial \mathbf{K}}{\partial \sigma_{L}} \mathbf{A}\right), \\
& \frac{\partial \mathbf{K}_{i, j}}{\partial \sigma_{L}}=\beta_{L} \frac{\partial}{\partial \sigma_{L}} k_{L}\left(\mathbf{x}_{i}, \mathbf{x}_{j}\right)=\beta_{L} \frac{2 \chi^{2}\left(\mathbf{L}_{i}, \mathbf{L}_{j}\right)}{\sigma_{L}^{3}} \exp \left(\frac{-\chi^{2}\left(\mathbf{L}_{i}, \mathbf{L}_{j}\right)}{\sigma_{L}^{2}}\right) .
\end{aligned}
$$

$\frac{\partial J}{\partial \sigma_{G}}$ can be computed similar to $\frac{\partial J}{\partial \sigma_{L}}$. Then we alternatively calculate $\mathbf{A}, \boldsymbol{\beta}$ and $\sigma$, which can be repeated until some predefined conditions are achieved. The detail algorithm is summarized as Algorithm 1.

Algorithm 1 Optimization algorithm for the fusing model.

\section{Require:}

The training set $\mathbf{X}$ and corresponding class label; Iteration times $T$; Iteration coefficient $\rho$. 


\section{Ensure:}

The projection matrix $\mathbf{A}$, the fusing weights $\beta$ and the kernel parameters $\sigma$.

1: Let $\boldsymbol{\beta}=[0.5,0.5]^{T}$ and $\boldsymbol{\sigma}$ be the mean distance of all pairs of training samples.

2: Optimize A according to Eq. (9).

3: Optimize $\beta$ according to Eq. (10).

4: Optimize $\sigma$ according to Eq. (13).

5: If $t<T$, set $t=t+1$, goto step 2 .

6: return $\mathrm{A}, \beta$ and $\sigma$.

\subsection{Complexity analysis}

In the training stage, optimizing the projection matrix $\mathbf{A}$ by Eq. (9) is an eigen-decomposition problem. The classical solution algorithm requires $O\left(N^{3}\right)$ operations $[20,24]$, where $N$ is the number of training samples. However, Cai et al. presented an algorithm for KDA, called Spectral Regression Kernel Discriminant Analysis (SRKDA) [24], and designed an incremental SRKDA to solve the eigen-decomposition problem, which only requires $O\left(N^{2}\right)$ operations. Optimizing the fusing weights $\beta$ by Eq. (11) requires $O\left(n^{2} m\right)$ operations, where $n$ is the number of variables and $m$ is the number of constraints, $n$ is linear to the number of the fusing descriptors, and $m$ is quadratic to that one. Optimizing the kernel parameters $\sigma$ by Eq. (13) only needs to calculate the gradient direction once. Because we only consider two kinds of descriptors, the computational complexity focuses on solving eigen-decomposition problem Eq. (9).

In the testing stage, we first need to project the testing sample by KDA, which requires $O(N d)$ operations, where $N$ is the number of training samples and $d$ is the feature dimension. Then we use nearest neighbor classifier to classify the 
testing sample, which needs $O(N p)$ operations, where $p$ is the reduced feature dimension by KDA. In summary, the testing process only requires $O(N)$ operations.

Because the training process requires $O\left(N^{2}\right)$ operations, and the testing process only requires $O(N)$ operations, the proposed method is scalable on large datasets. Especially, the testing process of the proposed method is much faster than some sparse representation-based classifiers, which will be proved in the experiment.

\section{Experimental results}

In this section, we perform experiments on three datasets: Labeled Faces in the Wild (LFW) [25], AR [26] and Extended Yale B [27], to demonstrate the performance of the proposed method. In Section 3.1, we present the results for face identification on LFW dataset. We first compare the proposed method with some state-of-the-art methods. Then the Gabor-based methods and the LBP-based methods are also compared with the proposed method. Last, we give the role of each step of the proposed method. In Section 3.2, the results of face verification under unrestricted protocol are presented. Finally, we give the results for AR database and Extended Yale B database in Section 3.3 and Section 3.4 respectively.

The proposed method Fuses Landmark-based LBP feature and Gabor feature at kernel level, thus we call it FLLG.

\subsection{Face identification on $L F W$ dataset}

LFW dataset contains 13233 images of 5749 people downloaded from the we$\mathrm{b}$, which is designed for unconstrained face recognition with dramatic variations 


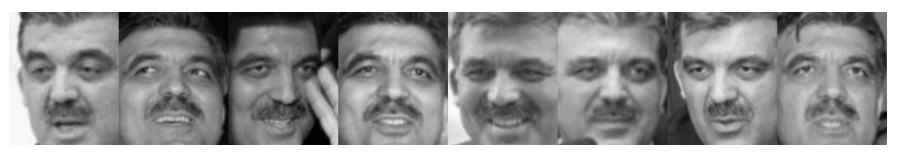

Figure 3: Some examples of one person in LFW dataset.

of pose, illumination, expression, misalignment and occlusion, and so on. Some examples of one person are shown in Fig. 3. As [10], a subset of LFW are used in the identification experiments, which consists of 5425 images of 311 subjects with no less than 6 samples per subject. We randomly select 5 samples per subject for training, and the rest for testing. We repeat the experiments 10 times, and report some statistics such as the mean accuracy and standard deviation.

\subsubsection{Comparison of state-of-the-art methods}

We compare the proposed method with some state-of-the-art methods, such as Gradientface[15], SRC[28], ESRC[29], CESR[30], SLF-RKR[10], MOST[4] and Joint Bayesian (JB)[21], and we will see that the performance of the proposed method is much better than the state-of-the-art methods. For SRC and ESRC, we first project the images to a PCA subspace with 1000 dimensions for the sake of computation efficiency, while almost not losing any useful information. The Dual Augmented Lagrange Multiplier(DALM)[31] method is used to solve all the $l_{1}$ minimization problems, where the sparsity coefficient $\lambda=0.001$. For JB, we also use the HDLBP feature[19] and project the features to a PCA subspace with 400 dimensions proposed in the original paper. When we increase the PCA dimensions to 1000 , the performance of JB is decreased dramatically. For Gradientface, we use the Cosine function to calculate the similarity between Gradientfaces. For MOST, we use the Euclidean distance for the extracted features. For SLF-RKR, we use the default optimal parameters in the original paper. For the proposed 
fusing model, we simply let iteration times $T=10$ and iteration coefficient $\rho$ be the mean distance of all pairs of training samples.

Fig. 4 shows the boxplot of the identification accuracies for state-of-the-art methods and the proposed method on LFW dataset. The box has lines at the lower quartile, median, and upper values of 10 recognition accuracies. The whiskers are lines extending from each end of the box to show the extent of the rest of the data. According to the results, we can draw the following conclusions:

- Gradientface, SRC, ESRC and CESR all get poor identification performance on the database, with the mean identification accuracies of 27.89 , $38.77,45.90$ and 36.09 percent, respectively.

- SLF-RKR is a LBP-based kernel method, whose performance is better than SRC and ESRC. MOST is based on Gabor feature and multiple kernel learning, and get better result.

- HDLBP+JB is also get good performance with a mean accuracy of 84.62 percent, which has shown very good result for face verification[19]. We will see in next sub-section that JB only get a mean accuracy of 67.61 percent based on gird-based LBP feature, which is even much lower than KDA that gets 76.19 percent. It proves that JB can't get as good result for face identification as that for verification.

- The proposed FLLG significantly improves the performance compared with the other state-of-the-art methods, geting a mean accuracy of 89.57 percent, which clearly shows the effectiveness of the proposed method in dealing with uncontrolled face identification. 


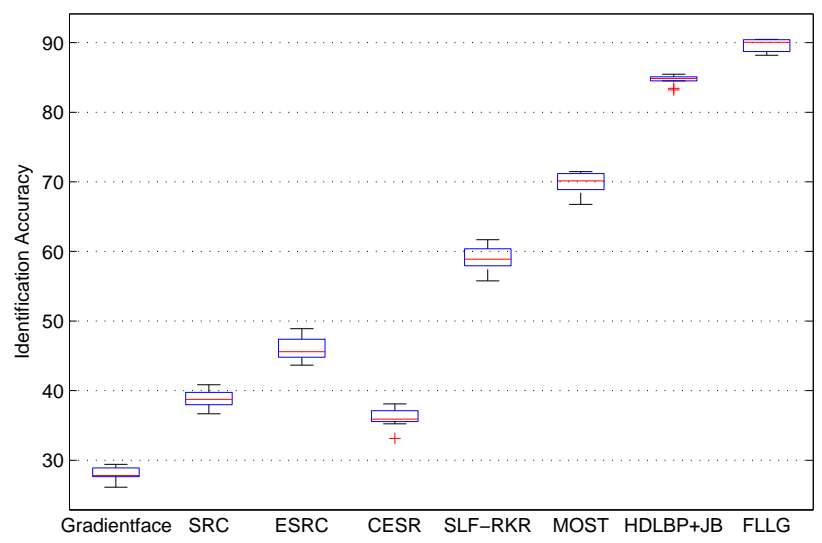

Figure 4: Comparison of state-of-the-art methods and the proposed method on LFW dataset.

Table 1: Comparison of training time and classification time for state-of-the-art methods.

\begin{tabular}{ccrc}
\hline Methods & $\begin{array}{c}\text { Training } \\
\text { time(s) }\end{array}$ & $\begin{array}{r}\text { Classification } \\
\text { time(s) }\end{array}$ & $\begin{array}{c}\text { Mean } \\
\text { accuracy(\%) }\end{array}$ \\
\hline SRC & - & 2.2 & 38.77 \\
ESRC & - & 7.9 & 45.90 \\
CESR & - & 24.1 & 36.09 \\
SLF-RKR & - & 18.2 & 58.99 \\
MOST & 490 & 0.20 & 69.78 \\
HDLBP+JB & 580 & 0.22 & 84.62 \\
FLLG & 672 & 0.28 & 89.57 \\
\hline
\end{tabular}


Table 1 shows the comparison of training time and classification time for state-of-the-art methods. The recognition accuracy is the mean value of 10 random experiments. We can find that the sparse representation-based classifiers such as SRC, ESRC, CESR and SLF-RKR do not have any training process, but they take much more classification time, and get lower recognition accuracies. The classification of MOST is the fastest, but its recognition accuracy is not good enough. The classification time of FLLG is only a little more than HDLBP+JB, and the recognition accuracy of FLLG is the highest.

\subsubsection{Comparison of Gabor-based methods}

Because the proposed method uses Gabor feature, we compare it with some other Gabor-based methods, such as 1NN, PCA, LDA, SRC, KDA, JB. We use the grid-based Gabor feature for these methods. For PCA, we report the accuracy at 1000 dimensions. We first project the Gabor feature to a PCA subspace with 1000 dimensions for LDA and SRC, and 400 dimensions for JB.

Fig. 5 shows the Comparison of the Gabor-based methods and the proposed method on LFW dataset. According to the results, we can draw the following conclusions:

- $1 \mathrm{NN}$ and PCA get very low identification accuracies, because there are dramatic intra-class variations on LFW database though based on Gabor feature. The performance of LDA is better than PCA because LDA is a supervised method, but it is still poor.

- Based on Gabor feature, SRC is significantly better than the above methods and the one based on original image pixel, which demonstrates Gabor feature is good feature for face recognition, especially using SRC. 
- The performance of JB is better than PCA and LDA, but is worse than S$\mathrm{RC}$, which shows that though the binary classification method can get high performance for face verification, but it can't get high performance for face identification.

- The performance of the proposed FLLG is much better than all other methods, which clearly shows the superiority of the proposed method.

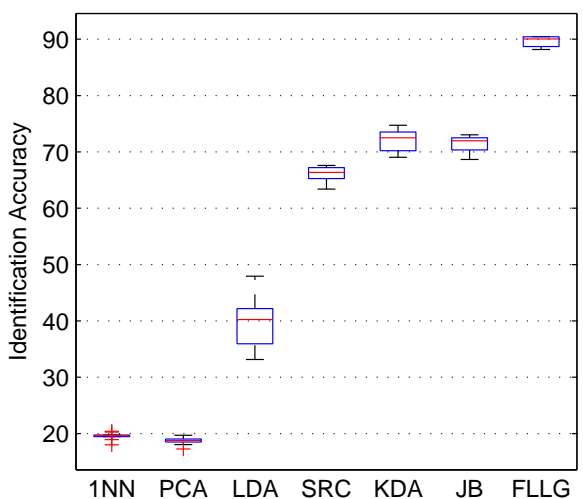

Figure 5: Comparison of Gabor-based methods and the proposed method on LFW dataset.

\subsubsection{Comparison of LBP-based methods}

Similar to the comparison of Gabor-based methods, we compare the proposed method with some traditional LBP-based methods. Besides the methods used in the above subsection, we also use the chi-square distance for $1 \mathrm{NN}$, denoted by $1 \mathrm{NN}(\mathrm{X} 2)$, and the RBF kernel with chi-square distance for KDA, denoted by $\mathrm{KDA}(\mathrm{X} 2)$, to further exploit the advantage of LBP feature.

Fig. 6 shows the boxplot of the identification accuracies for the LBP-based methods on LFW dataset. As we can see, the relation of the results for $1 \mathrm{NN}$, 
PCA, LDA, SRC, KDA, JB and the proposed method is similar to those in Fig. 5. The identification accuracies of $1 \mathrm{NN}(\mathrm{X} 2)$ and $\mathrm{KDA}(\mathrm{X} 2)$ are significantly better than 1NN and KDA, respectively, which shows that the chi-square distance is better than $l_{2}$ norm distance for LBP feature. The performance of KDA is much better than SRC and JB by selecting an appropriate kernel, which proves that the kernel based method can efficiently model the highly non-linear problem for uncontrolled face recognition.

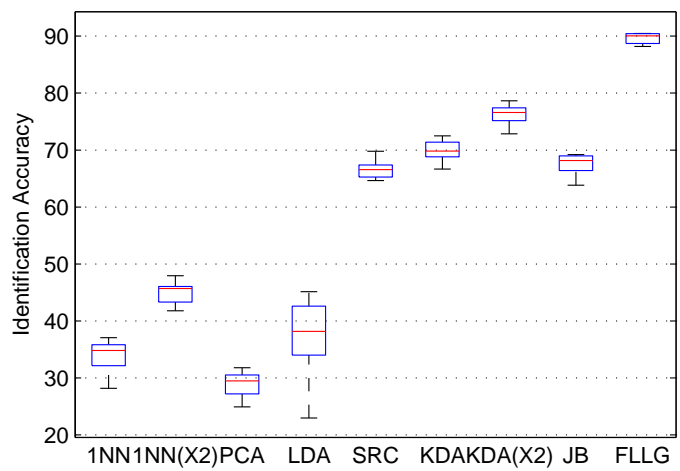

Figure 6: Comparison of LBP-based methods and the proposed method on LFW dataset.

\subsubsection{The role of each step of the proposed method}

Each step of the proposed method make a contribution to the high performance. Table 2 shows the role of each step of the proposed method. According to the results, we can draw the following conclusions:

- The identification accuracy of the grid-based multi-scale LBP feature is higher than grid-based LBP feature with only one scale, and that of landmarkbased Multi-scale LBP is better than landmark-based LBP too, which proves that the multi-scale feature can improve the performance, especially for the landmark-based feature. 
Table 2: The role of each step of the proposed method.

\begin{tabular}{ll}
\hline Method & Mean acc. \pm std. dev. \\
\hline Grid-based LBP & $76.19 \pm 2.0$ \\
Grid-based multi-scale LBP & $81.27 \pm 1.3$ \\
Landmark-based LBP & $80.44 \pm 1.8$ \\
Landmark-based multi-scale LBP & $86.12 \pm 1.5$ \\
Grid-landmark-based multi-scale LBP & $86.69 \pm 1.4$ \\
& \\
Grid-based Gabor & $72.24 \pm 1.6$ \\
Landmark-based Gabor & $81.77 \pm 1.7$ \\
Grid-landmark-based Gabor & $82.12 \pm 1.8$ \\
& \\
HDLBP & $83.59 \pm 1.1$ \\
FLLG & \\
FLLG & \\
FLLG & $\mathbf{8 7 . 8 9} \pm \mathbf{1 . 0}$ \\
\hline
\end{tabular}


- The landmark-based features significantly outperform the grid-based features, no matter using LBP or Gabor feature, which shows that the landmarkbased features are good at misaligned condition or pose variation on uncontrolled datasets.

- Simply combining the grid-based and landmark-based multi-scale LBP at the feature level, i.e., Grid-landmark-based multi-scale LBP or Gabor feature, we can get higher performance, because the grid-based feature can provide the geometric information among the landmarks.

- Our landmark-based multi-scale LBP feature outperforms HDLBP, though the dimension of HDLBP is much higher than that of our feature. It proves the effectiveness of our feature extraction method.

- Fusing the LBP feature and the Gabor feature by the proposed kernel-level fusing model, FLLG gets the highest performance. It increases about an average of 8.3 percent than grid-based multi-scale LBP, 3.4 percent than landmark-based multi-scale LBP, 7.4 percent than Grid-landmark-based Gabor, and 6.0 percent than HDLBP feature, which clearly shows the proposed method can effectively fuse the LBP and Gabor features.

To examine the contribution of each component of the proposed fusing model, we also consider the following two situations. In the first case, denoted as FLLG ${ }^{\sigma}$, the standard deviations are not automatically learnt, i.e., we simply let the standard deviation be the mean distance of training samples and learn the weighted coefficients between kernels and the projection matrix. In the second case, denoted as $\mathrm{FLLG}^{\beta}$, the weighted coefficients between kernels are not automatically learnt, i.e., we simply set the same weighted coefficient for each kernel, and learn the 
Table 3: Comparison of different alignment methods for grid-based feature.

\begin{tabular}{ccccc}
\hline Methods & Original & VGG & TCDCN & SDM \\
\hline Mean acc. \pm std. dev. & $67.03 \pm 1.9$ & $74.43 \pm 2.2$ & $75.12 \pm 2.3$ & $76.19 \pm 2.0$ \\
\hline
\end{tabular}

projection matrix and the standard deviations. We can find that the performance of FLLG is significantly better than that of $\mathrm{FLLG}^{\sigma}$, which proves the efficiency of the learning model for the standard deviation. FLLG outperforms FLLG ${ }^{\beta}$ too, which shows the necessity of the learning model for the weighted coefficients.

\subsubsection{Comparison of different alignment methods for grid-based feature.}

For grid-based methods, the features in the same location of two images are compared with each other, so the face images require to be aligned in a canonical space as accurately as possible. There are many methods to align images, such as Oxford Visual Geometry Group baseline face processing (VGG) [32], Supervised Descent Method (SDM) [23] and Tasks-Constrained Deep Convolutional Network (TCDCN) [33]. Table 3 shows the comparison of different alignment methods for grid-based feature, where the recognition accuracy is the mean value of 10 random experiments, and the "Original" method uses the original LFW images provided by the official website. We find that VGG, TCDCN and SDM get similar results, and is much better than the original images.

Because the accuracy of SDM is the highest, we use SDM to align the original images. Specifically, for each image, we first run the Viola Jones face detector [34] to locate the face. Then we use SDM to detect the facial landmark positions. Finally, we apply similarity transformation using these landmark points to transform a face to a canonical frame, and crop a center region of size $120 \times 100$ for 


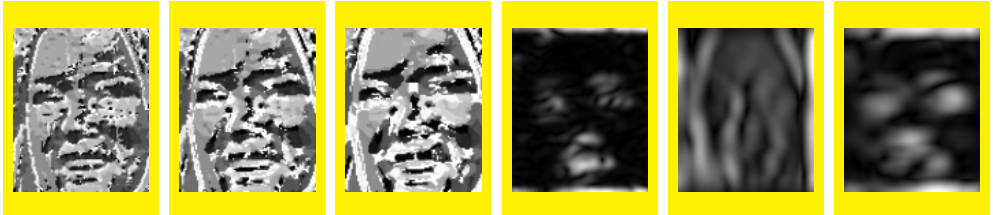

Figure 7: LBP features and Gabor features. The first three images are the LBP features with different scales, and the last three images are the Gabor features with different scales and orientations. We can find that LBP feature can encode fine details of facial appearance and texture, and Gabor feature can reflect facial shape and appearance information. Their complementary nature makes them good candidates for fusion.

further feature extraction.

\subsubsection{Comparison of fusing different kinds of features.}

For the proposed fusing model, selecting appropriate and complementary component features is crucial for good performance. LBP feature is a good choice for coding fine details of facial appearance and texture. In contrast, Gabor feature can encode facial shape and appearance information. Both representations can deal with illumination. Their complementary nature makes them good candidates for fusion, as shown in Fig. 7.

Besides LBP feature and Gabor feature, we can fuse more features. Recently, some learning-based features, such as LEarning-based descriptor (LE) [18] and Discriminant Face Descriptor (DFD) [1], can improve the classification results. Here we consider fusing more features, including the learning-based features and SIFT feature [16]. Table 4 shows the comparison of fusing different kinds of features, where the SIFT, LE and DFD features are created with multi-scale and landmark-based type, just like the proposed landmark-based multi-scale LBP feature. We can find that when fusing two types of features, LBP+Gabor gets better performance than LBP+SIFT, LBP+LE and LBP+DFD. When fusing more than 
Table 4: Comparison of fusing different kinds of features.

\begin{tabular}{cc}
\hline Features & Mean acc. \pm std. dev. \\
\hline LBP+Gabor & $89.57 \pm 1.0$ \\
LBP+SIFT & $88.18 \pm 1.1$ \\
LBP+LE & $87.64 \pm 1.2$ \\
LBP+DFD & $87.56 \pm 1.3$ \\
LBP+Gabor+SIFT & $89.59 \pm 1.1$ \\
LBP+Gabor+LE & $89.53 \pm 1.2$ \\
LBP+Gabor+DFD & $89.32 \pm 1.1$ \\
LBP+Gabor+SIFT+DFD & $89.61 \pm 1.1$ \\
\hline
\end{tabular}

two types of features, the recognition accuracy cannot be significantly improved compared with LBP+Gabor, but much computational cost is required. So we only consider to fuse LBP feature and Gabor feature for face recognition.

In recent years, deep learning has been proved to be able to learn the natural patterns in images. We also combine these deep learning-based features with the proposed fusing model to improve the performance. Table 5 shows the performance of combining two deep learning-based features, i.e., DeepID2 [35] and GoogleNet [36], with the proposed fusing model. We can find that combining DeepID2 and GoogleNet with the proposed fusing model can improve the recognition accuracy.

\subsection{Face verification on $L F W$ dataset}

LFW specifies a number of evaluation protocols for face verification. We follow the unrestricted protocol and only use training data provided by LFW. There 
Table 5: Combining deep learning-based features with the proposed fusing model.

\begin{tabular}{cc}
\hline Features & Mean acc. \pm std. dev. \\
\hline DeepID2 & $98.55 \pm 0.3$ \\
GoogleNet & $98.58 \pm 0.2$ \\
DeepID2+GoogleNet & $99.05 \pm 0.2$ \\
\hline
\end{tabular}

are more than 10000 training samples of more than 5000 people, but most of people have only one or two samples. For the proposed method, to learn more representative features, we select the top 300 persons which have the most number of images, where there are more than 4000 samples, to train the projection matrix $\mathbf{A}$ and the fusing weights $\beta$. Then we project all training samples into the learned space of 299 dimensions. In order to get better performance for face verification, we use ITML[37] to learning a Mahalanobis distance function for the binary-class problem in the projected space of lower dimension, where 10000 positive pairs and 10000 negative pairs are uniformly sampled to learn the Mahalanobis matrix.

We compare against the best results listed in the LFW official web-site, such as Combined multishot [38], LBP multishot [38], LBP PLDA [39], Joint Bayesian [21], HDLBP+JB [19], Fisher vector [40], ConvNet-RBM [41], SLBP [42], VisionLabs [43], Aurora [44] and HPEN + HDLBP + JB [45], and the deep learningbased methods with outside training data such as ConvNet-RBM [41], DeepFace [46], DeepID [47], DeepID2 [35] and DeepID3 [48].

Table 6 lists mean verification accuracies and standard deviations for stateof-the-art methods on LFW dataset under unrestricted protocol without outside 
training data. Our method achieves $94.03 \%$ face verification accuracy, which is better than HDLBP+JB [19] and Fisher vector [40], and is only lower than $\mathrm{H}$ PEN+HDLBP+JB without outside training data.

Though the performances of the deep learning-based methods are better than the proposed method, they come with some challenges. First, it remains unclear how to design a "good" CNN architecture to adapt to a specific classification task due to the lack of theoretical guidance [49]. Second, the training data is very important for the performance of face recognition. To achieve better results, we need to add more faces which are collected in the same situation as the evaluation dataset [50]. Finally, because of the requirement for large amounts of training data, it needs high computational cost during the training process [51]. So our method which is less costly to train and evaluate is still competitive for face verification.

Fig. 8 shows the ROC curves averaged over 10 fold cross validation without outside training data. For more explicit, we only show the figure where false positive rate is from 0 to 0.5 and true positive rate is from 0.5 to 1 . It can be found that the performance of our method is as good as the best results, which demonstrates that our method can not only get good identification performance but also do well in verification task.

\subsection{Face identification on AR database}

In this section, we perform experiments on AR face databases, and compare the proposed method with several state-of-the-art methods, such as LDA[22], Gradientface (GF)[15], SRC[28], ESRC[29], SLF-RKR[10], MOST[4] and JB[21].

The AR face database[26] consists of over 4,000 facial images from 126 subjects (70 men and 56 women). These images suffer different facial variations, 
Table 6: Mean verification accuracies and standard deviations for state-of-the-art methods on LFW dataset under unrestricted protocol.

\begin{tabular}{|c|c|c|}
\hline & Method & Mean acc. \pm std. dev. \\
\hline \multirow{12}{*}{$\begin{array}{l}\text { without } \\
\text { outside } \\
\text { training } \\
\text { data }\end{array}$} & Combined multishot & $89.50 \pm 0.51$ \\
\hline & LBP multishot & $85.17 \pm 0.61$ \\
\hline & LBP PLDA & $87.33 \pm 0.55$ \\
\hline & Joint Bayesian & $90.90 \pm 1.48$ \\
\hline & HDLBP+JB & $93.18 \pm 1.07$ \\
\hline & Fisher vector & $93.03 \pm 1.05$ \\
\hline & ConvNet-RBM & $91.75 \pm 0.48$ \\
\hline & SLBP & $90.00 \pm 1.33$ \\
\hline & VisionLabs & $92.90 \pm 0.31$ \\
\hline & Aurora & $93.24 \pm 0.44$ \\
\hline & $\mathrm{HPEN}+\mathrm{HDLBP}+\mathrm{JB}$ & $94.87 \pm 0.38$ \\
\hline & FLLG & $94.03 \pm 1.16$ \\
\hline \multirow{5}{*}{$\begin{array}{l}\text { with } \\
\text { outside } \\
\text { training } \\
\text { data }\end{array}$} & ConvNet-RBM & $92.52 \pm 0.38$ \\
\hline & DeepFace & $97.35 \pm 0.25$ \\
\hline & DeepID & $97.45 \pm 0.26$ \\
\hline & DeepID2 & $99.15 \pm 0.13$ \\
\hline & DeepID3 & $99.53 \pm 0.10$ \\
\hline
\end{tabular}




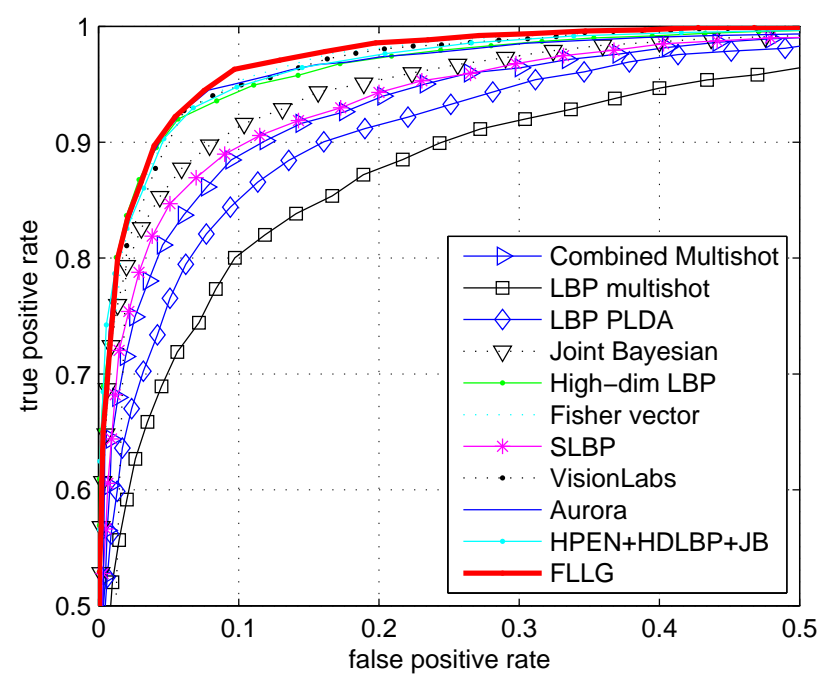

Figure 8: ROC curves averaged over 10 fold cross validation under LFW unrestricted protocol without outside training data.

including various facial expressions, illumination variations and occlusion by sunglasses or scarf. In our experiment, we use a subset consisting 119 subjects. For each subject, 14 images with only illumination changes and expressions are selected and taken into two sessions. The gray-scale images are cropped and resized to $112 \times 92$.

To ensure that our results will not depend on any special choice of the training data, for each subject, seven images from the two sessions are randomly selected for training and the rest seven images were for testing. We repeat the experiment 10 times. Fig. 9 shows the boxplot of the identification accuracies for state-ofthe-art methods on AR dataset. It can be found that the proposed method achieves the best result with a mean of 98.76 percent, which is $8.8,22.9,5.4,2.4,2.5,0.2$ and 5.3 percent higher than those of LDA, Gradientface, SRC, ESRC, SLF-RKR, MOST and JB, respectively. The standard deviation of FLLG is the smallest, 
which shows the stability of the proposed method.

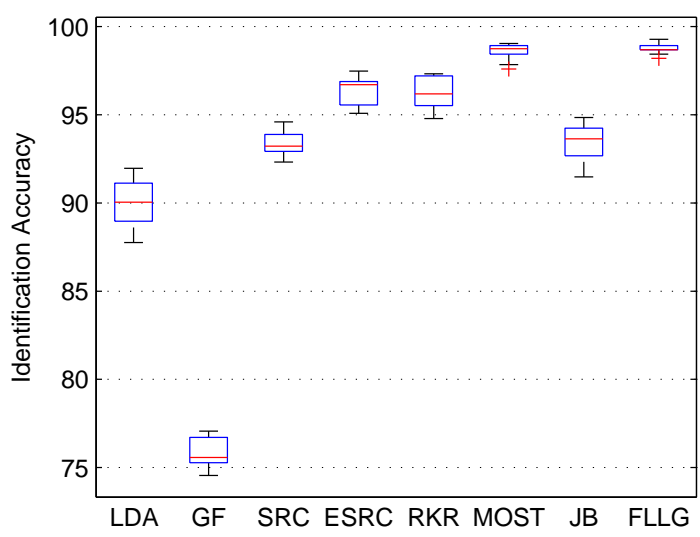

Figure 9: Boxplot of the identification accuracies for state-of-the-art methods on AR dataset.

\subsection{Face identification on Extended Yale B database}

In this section, we perform experiments on Extended Yale B face databases, and compare the proposed method with several state-of-the-art methods. Extended Yale B database[27] contains about 2,414 frontal face images 38 individuals. We use the cropped and normalized $96 \times 84$ face images, which are taken under extreme illumination variation. For each subject, we randomly select some images for training respectively, and the rest for testing. We repeat the experiment 10 times in each condition.

Fig. 10 shows the boxplot of the identification accuracies for state-of-the-art methods on Extended Yale B dataset with 5 samples per person. It can be found that Gradientface gets high performance on the dataset with extreme illumination variation. The proposed method achieves the best result with a mean of 98.79 percent, which is $4.7,6.5,12.1,5.1,8.2,6.1$ and 14.5 percent higher than those of LDA, Gradientface, SRC, ESRC, SLF-RKR, MOST and JB, respectively. 


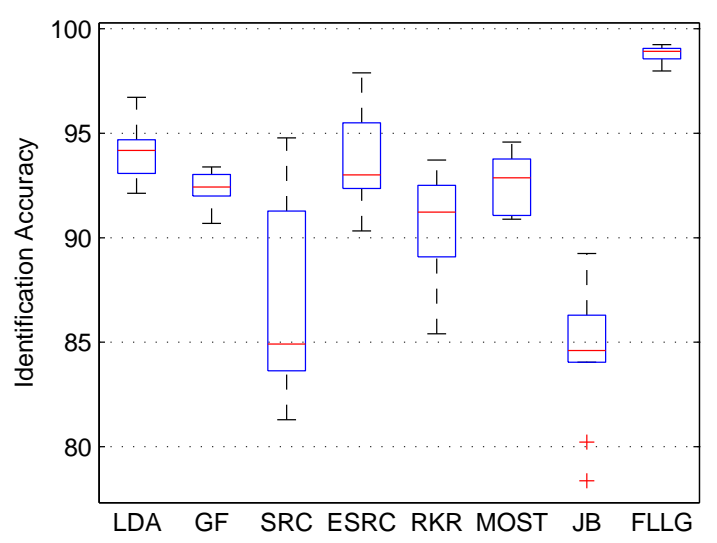

Figure 10: Boxplot of the identification accuracies for state-of-the-art methods on Extended Yale B dataset with 5 samples per person.

\section{Conclusion}

In this paper, we propose an improved landmark-based feature and a novel fusing model for face recognition that can not only get good identification performance but also do well in verification task. The proposed landmark-based multiscale LBP feature samples features around landmarks instead of fixed grid and adopts multi-scale histogram to get more robust representation. The multi-scale fixed grid sampling features are also combined, which can provide the geometric information among the landmarks. The proposed fusing model fuses LBP feature and Gabor feature at kernel-level, where the weighted coefficients between kernels, the discriminant projection matrix and the standard deviations of RBF kernel are simultaneously learnt. To solve the kernel-level fusing model, an optimization algorithm is also proposed, which alternatively calculates the three types of parameters. Experiments are done on several datasets, giving very promising results for the proposed method in both identification and verification. 


\section{References}

[1] Z. Lei, M. Pietikainen, S. Z. Li, Learning discriminant face descriptor, IEEE Transactions on Pattern Analysis and Machine Intelligence 36 (2) (2014) 289-302.

[2] X. Y. Tan, B. Triggs, Fusing Gabor and LBP feature sets for kernel-based face recognition, in: IEEE International Workshop on Analysis and Modeling of Faces and Gestures (AMFG), 2007, pp. 235-249.

[3] Y. Y. Lin, T. L. Liu, C. S. Fuh, Multiple kernel learning for dimensionality reduction, IEEE Transactions on Pattern Analysis and Machine Intelligence 33 (6) (2011) 1147-1160.

[4] C. X. Ren, D. Q. Dai, X. X. Li, Z. R. Lai, Band-reweighed gabor kernel embedding for face image representation and recognition, IEEE Transactions on Image Processing 23 (2) (2014) 725-740.

[5] T. Ojala, M. Pietikäinen, D. Harwood, A comparative study of texture measures with classification based on featured distributions, Pattern Recognition 29 (1) (1996) 51-59.

[6] T. Ojala, M. Pietikainen, T. Maenpaa, Multiresolution gray-scale and rotation invariant texture classification with local binary patterns, IEEE Transactions on Pattern Analysis and Machine Intelligence 24 (7) (2002) 971-987.

[7] T. Ahonen, A. Hadid, M. Pietikainen, Face description with local binary patterns: Application to face recognition, IEEE Transactions on Pattern Analysis and Machine Intelligence 28 (12) (2006) 2037-2041. 
[8] K. K. Huang, D. Q. Dai, C. X. Ren, Z. R. Lai, Learning kernel extended dictionary for face recognition, IEEE Transactions on Neural Networks and Learning Systems, preprint.

[9] S. C. Liao, X. X. Zhu, Z. Lei, L. Zhang, S. Z. Li, Learning multi-scale block local binary patterns for face recognition, in: Advances in Biometrics, Springer, 2007, pp. 828-837.

[10] M. Yang, L. Zhang, S. C. K. Shiu, D. Zhang, Robust kernel representation with statistical local features for face recognition, IEEE Transactions on Neural Networks and Learning Systems 24 (6) (2013) 900-912.

[11] C. J. Liu, H. Wechsler, Gabor feature based classification using the enhanced fisher linear discriminant model for face recognition, IEEE Transactions on Image Processing 11 (4) (2002) 467-476.

[12] L. L. Shen, L. Bai, M. Fairhurst, Gabor wavelets and general discriminant analysis for face identification and verification, Image and Vision Computing 25 (5) (2007) 553-563.

[13] B. C. Zhang, S. G. Shan, X. L. Chen, W. Gao, Histogram of gabor phase patterns: A novel object representation approach for face recognition, IEEE Transactions on Image Processing 16 (1) (2007) 57-68.

[14] S. F. Xie, S. G. Shan, X. L. Chen, J. Chen, Fusing local patterns of gabor magnitude and phase for face recognition, IEEE Transactions on Image Processing 19 (5) (2010) 1349-1361.

[15] T. P. Zhang, Y. Y. Tang, B. Fang, Z. W. Shang, X. Y. Liu, Face recognition 
under varying illumination using gradientfaces, IEEE Transactions on Image Processing 18 (11) (2009) 2599-2605.

[16] D. G. Lowe, Distinctive image features from scale-invariant keypoints, International Journal of Computer Vision 60 (2) (2004) 91-110.

[17] N. Dalal, B. Triggs, Histograms of oriented gradients for human detection, in: IEEE Conference Computer Vision and Pattern Recognition (CVPR), 2005, pp. 886-893.

[18] Z. M. Cao, Q. Yin, X. O. Tang, J. Sun, Face recognition with learning-based descriptor, in: IEEE Conference on Computer Vision and Pattern Recognition (CVPR), 2010, pp. 2707-2714.

[19] D. Chen, X. D. Cao, F. Wen, J. Sun, Blessing of dimensionality: Highdimensional feature and its efficient compression for face verification, in: IEEE Conference on Computer Vision and Pattern Recognition (CVPR), 2013, pp. 3025-3032.

[20] G. Baudat, F. Anouar, Generalized discriminant analysis using a kernel approach, Neural Computation 12 (10) (2000) 2385-2404.

[21] D. Chen, X. D. Cao, L. W. Wang, F. Wen, J. Sun, Bayesian face revisited: A joint formulation, in: European Conference on Computer Vision(ECCV), 2012, pp. 566-579.

[22] P. N. Belhumeur, J. P. Hespanha, D. J. Kriegman, Eigenfaces vs. fisherfaces: Recognition using class specific linear projection, IEEE Transactions on Pattern Analysis and Machine Intelligence 19 (7) (1997) 711-720. 
[23] X. H. Xiong, F. D. L. Torre, Supervised descent method and its applications to face alignment, in: IEEE Conference on Computer Vision and Pattern Recognition (CVPR), 2013, pp. 532-539.

[24] D. Cai, X. He, J. Han, Speed up kernel discriminant analysis, The VLDB Journal 20 (1) (2011) 21-33.

[25] G. B. Huang, M. Ramesh, T. Berg, E. Learned-Miller, Labeled faces in the wild: A database for studying face recognition in unconstrained environments, Tech. Rep. 07-49, University of Massachusetts, Amherst (Oct 2007).

[26] A. M. Martinez, R. Benavente, The AR face database, Tech. rep., CVC \#24 (June 1998).

[27] A. Georghiades, P. Belhumeur, D. Kriegman, From few to many: Illumination cone models for face recognition under variable lighting and pose, IEEE Transactions on Pattern Analysis and Machine Intelligence 23 (6) (2001) 643-660.

[28] J. Wright, A. Y. Yang, A. Ganesh, S. S. Sastry, Y. Ma, Robust face recognition via sparse representation, IEEE Transactions on Pattern Analysis and Machine Intelligence 31 (2) (2009) 210-227.

[29] W. H. Deng, J. N. Hu, J. Guo, Extended SRC: Undersampled face recognition via intraclass variant dictionary, IEEE Transactions on Pattern Analysis and Machine Intelligence 34 (9) (2012) 1864-1870.

[30] R. He, W. S. Zheng, B. G. Hu, Maximum correntropy criterion for robust face recognition, IEEE Transactions on Pattern Analysis and Machine Intelligence 33 (8) (2011) 1561-1576. 
[31] A. Y. Yang, Z. H. Zhou, A. G. Balasubramanian, S. S. Sastry, Y. Ma, Fast $l_{1}$-minimization algorithms for robust face recognition, IEEE Transactions on Image Processing 22 (8) (2013) 3234-3246.

[32] M. Everingham, J. Sivic, A. Zisserman, Taking the bite out of automated naming of characters in tv video, Image and Vision Computing 27 (5) (2009) $545-559$.

[33] Z. Zhang, P. Luo, C. C. Loy, X. Tang, Learning deep representation for face alignment with auxiliary attributes, IEEE Transactions on Pattern Analysis and Machine Intelligence 38 (5) (2016) 918-930.

[34] P. Viola, M. Jones, Robust real-time object detection, International Journal of Computer Vision 57 (2) (2001) 137-154.

[35] Y. Sun, Y. Chen, X. Wang, X. Tang, Deep learning face representation by joint identification-verification, in: Advances in Neural Information Processing Systems (NIPS), 2014, pp. 1988-1996.

[36] C. Szegedy, W. Liu, Y. Jia, P. Sermanet, S. Reed, D. Anguelov, D. Erhan, V. Vanhoucke, A. Rabinovich, Going deeper with convolutions, in: IEEE Conference on Computer Vision and Pattern Recognition (CVPR), 2014, pp. $1-9$.

[37] J. Davis, B. Kulis, S. Sra, I. Dhillon, Information-theoretic metric learning, in: International Conference on Machine Learning(ICML), 2007, pp. 209_ 216.

[38] L. Wolf, T. Hassner, Y. Taigman, Effective unconstrained face recognition by combining multiple descriptors and learned background statistics, IEEE 
Transactions on Pattern Analysis and Machine Intelligence 33 (10) (2011) $1978-1990$.

[39] P. Li, Y. Fu, U. Mohammed, J. H. Elder, S. J. D. Prince, Probabilistic models for inference about identity, IEEE Transactions on Pattern Analysis and Machine Intelligence 34 (1) (2012) 144-157.

[40] K. Simonyan, O. M. Parkhi, A. Vedaldi, A. Zisserman, Fisher vector faces in the wild, in: British Machine Vision Conference(BMVC), 2013, pp. 7-19.

[41] Y. Sun, X. G. Wang, X. O. Tang, Hybrid deep learning for face verification, in: IEEE International Conference on Computer Vision (ICCV), 2013, pp. $1489-1496$.

[42] C. Huang, S. H. Zhu, K. Yu, Large scale strongly supervised ensemble metric learning, with applications to face verification and retrieval, Tech. rep., NEC TR115 (Oct 2011).

[43] VisionLabs, Visionlabs ver. 1.0, http://www.visionlabs.ru/face-recognition.

[44] T. Heseltine, P. Szeptycki, J. Gomes, M. C. Ruiz, P. Li, Evaluation of algorithm "Aurora-c-2014-1" on Labeled Faces in the Wild, Tech. rep., Aurora Computer Services Ltd (Jan 2014).

[45] X. Y. Zhu, Z. Lei, J. J. Yan, D. Yi, S. Z. Li, High-fidelity pose and expression normalization for face recognition in the wild, in: IEEE Conference on Computer Vision and Pattern Recognition (CVPR), 2015, pp. 787-796.

[46] Y. Taigman, M. Yang, M. Ranzato, L. Wolf, Deepface: Closing the gap to 
human-level performance in face verification, in: IEEE Conference Computer Vision and Pattern Recognition (CVPR), 2014, pp. 1701-1708.

[47] Y. Sun, X. G. Wang, X. O. Tang, Deep learning face representation from predicting 10,000 classes, in: IEEE Conference on Computer Vision and Pattern Recognition (CVPR), 2014, pp. 1891-1898.

[48] Y. Sun, D. Liang, X. G. Wang, X. O. Tang, Deepid3: Face recognition with very deep neural networks, arXiv preprint arXiv:1502.00873.

[49] G. S. Hu, Y. X. Yang, D. Yi, J. Kittler, W. Christmas, S. Z. Li, T. Hospedales, When face recognition meets with deep learning: an evaluation of convolutional neural networks for face recognition, arXiv preprint arXiv:1504.02351.

[50] J. G. Liu, Y. F. Deng, C. Huang, Targeting ultimate accuracy: Face recognition via deep embedding, arXiv preprint arXiv:1506.07310.

[51] F. Perronnin, D. Larlus, Fisher vectors meet neural networks: A hybrid classification architecture, in: IEEE Conference on Computer Vision and Pattern Recognition (CVPR), 2015, pp. 3743-3752. 